\title{
In vivo Characterization of MRI-based T1w/T2w Ratios and Covariance Network in Temporal Lobe Epilepsy
}

\author{
Yuchao Jiang, Wei Li, Yingjie Qin, Le Zhang, Xin Tong, Fenglai Xiao, Qiyong Gong, Dong Zhou, \\ Dongmei An, Cheng Luo, Dezhong Yao
}

\begin{abstract}
Temporal lobe epilepsy (TLE) is the most common type of intractable epilepsy in adults. A novel method based on the ratio of T1-weighted (T1w) and T2weighted (T2w) magnetic resonance images can investigate brain microstructural changes and how these regional changes interact with each other. This study estimated $\mathrm{T} 1 \mathrm{w} / \mathrm{T} 2 \mathrm{w}$ ratios in 42 left TLE (LTLE) and 42 right TLE (RTLE) patients and 41 healthy controls (HC). A T1w/T2w structural covariance network (SCN) was built by calculating correlations between any two regions across subjects and analysed by graph theory. Voxel-wise comparisons of T1w/T2w laterality were performed among the three groups. Compared to $\mathrm{HC}$, both patient groups showed decreased $\mathrm{T} 1 \mathrm{w} / \mathrm{T} 2 \mathrm{w}$ in frontotemporal regions, amygdala and thalamus; however, the LTLE showed lower T1w/T2w in left medial temporal regions than RTLE. Moreover, the LTLE exhibited decreased global efficiency compared with HC and more increased connections than RTLE. The laterality in putamen was differently altered between the two patient groups: higher laterality at posterior putamen in LTLE and higher laterality at anterior putamen in RTLE. This study demonstrated $\mathrm{T} 1 \mathrm{w} / \mathrm{T} 2 \mathrm{w}$ reductions in frontotemporal and subcortical regions and extensive disconnections of $\mathrm{SCN}$, providing evidence that TLE is a system disorder with widespread disruptions at regional and network levels. The putamen may play a transfer station role in damage spreading induced by epileptic seizures from the hippocampus.
\end{abstract}

Index Terms-Myelin; Temporal lobe epilepsy; Magnetic resonance image; Structural covariance; Putamen; Structural connectivity.

\section{INTRODUCTION}

$\mathrm{T}$ emporal lobe epilepsy (TLE) is the most common type of intractable epilepsy in adults [1]. Although magnetic resonance imaging (MRI) morphometric measures, such as cortical thickness or grey-matter volume, have demonstrated medial temporal atrophy, cortical thinning and subcortical abnormalities [2], a few studies found brain myelination alterations in TLE. For example, an earlier histopathological study of surgical specimens reported anomalies of cortical myelinated fibres in TLE [3]. A recent study has demonstrated atypical morphology of myelinated axons in TLE patients with temporo-polar blurring, using a combination of ex-vivo MRI and histological analysis [4]. To date, the non-invasive study of myelin changes in the human brain remains a challenge. Recently, a method based on the ratio of T1-weighted (T1w) and $\mathrm{T} 2$-weighted $(\mathrm{T} 2 \mathrm{w})$ magnetic resonance images provides insight in the characterization of microstructural myelination changes underlying brain cortical and subcortical grey-matter and white matter (WM) in vivo [5]. The $\mathrm{T} 1 \mathrm{w} / \mathrm{T} 2 \mathrm{w}$ ratio, by simultaneously enhancing the sensitivity to myelin signal intensity while reducing the inter-subject signal intensity bias, can be used as a measure for detecting changes in myelination degree associated with diseases [5]. T1w/T2w ratio has been widely applied to study cortical and WM changes in ageing adults, multiple sclerosis and post-traumatic stress disorder $[6$, 7].

It is still unclear how these regions with myelination changes interact with each other. Do structural alterations across brain regions synchronously co-change in patients with TLE? Are the topological properties of the structural covariance network distinct between TLE and healthy controls? Although accumulated evidence has suggested different damages of greymatter volume [8], cortical thickness [8], and the structural and functional network [9] between left TLE and right TLE, no studies have examined the impact of the seizure side on the myelination. As the most common drug-resistant type of epilepsy, TLE patients frequently receive an anterior temporal lobectomy (ATL) [1]. However, it remains unclear whether the myelination levels in the damaged regions before surgery improve after ATL.

This study applied the $\mathrm{T} 1 \mathrm{w} / \mathrm{T} 2 \mathrm{w}$ analysis workflow to investigate in vivo the microstructural feature of brain tissue in

This work was partly supported by the grant from the National Key R\&D Program of China (No. 2018YFA0701400), grants from the National Natural Science Foundation of China (No. grant number: 61933003, 81771402, 81771822, 81861128001, and 81771925), the CAMS Innovation Fund for Medical Sciences (CIFMS) (No. 2019-I2M-5-039) and the Project of Science and Technology Department of Sichuan Province (No. 2019YJ0179).

Y. Jiang, C. Luo and D. Yao are with the Clinical Hospital of Chengdu Brain Science Institute, MOE Key Lab for Neuroinformation, Center for Information in Medicine, High-Field Magnetic Resonance Brain Imaging Key Laboratory of Sichuan Province, School of life Science and technology, University of Electronic Science and Technology of China, Chengdu, 610054, P. R. China. (e-mail: chengluo@uestc.edu.cn)

W. Li, Y. Qin, L. Zhang, X. Tong, F. Xiao, D, Zhou and D. An are with the Department of Neurology, West China Hospital, Sichuan University, Chengdu, 610041, P. R. China.

Q. Gong is with the Huaxi MR Research Center, Department of Radiology, West China Hospital, Sichuan University, Chengdu, 610041, P. R. China. 
medRxiv preprint doi: https://doi.org/10.1101/2020.12.03.20243238; this version posted December 4, 2020. The copyright holder for this preprint (which was not certified by peer review) is the author/funder, who has granted medRxiv a license to display the preprint in perpetuity. It is made available under a CC-BY-NC-ND 4.0 International license .

a cohort of patients with left TLE, right TLE and healthy controls. Subsequently, we employed region of interest (ROI) analysis for the ROI-wise estimation of $\mathrm{T} 1 \mathrm{w} / \mathrm{T} 2 \mathrm{w}$ in cortical grey-matter, subcortical grey-matter and WM. Simultaneously, to evaluate the synchronous changes of $\mathrm{T} 1 \mathrm{w} / \mathrm{T} 2 \mathrm{w}$ across ROIs, we built a structural covariance network by calculating the correlations between any two ROIs across subjects in each group. We compared differences in the connections and topological properties of the structural covariance network among the left TLE, right TLE and healthy controls. In addition, we estimated the laterality map of $\mathrm{T} 1 \mathrm{w} / \mathrm{T} 2 \mathrm{w}$ for homotopic voxels, which can achieve a voxel-wise comparison of T1w/T2w laterality among the left TLE, right TLE and healthy subjects. Finally, we investigated the longitudinal changes of T1w/T2 w using another longitudinal sample including postATL TLE patients.

\section{DATA}

\section{A. Participants}

This study included forty-two left TLE patients (LTLE group: 15 females, Age=25.29 \pm 7.96 ), 42 right TLE (RTLE group: 18 females, Age $=26.96 \pm 8.33$ ) and 41 healthy controls (HC group, 25 females, Age $=29.37 \pm 10.63$ ). All subjects were recruited from the West China Hospital and scanned by the MRI. All patients were diagnosed with unilateral TLE according to the International League Against Epilepsy (ILAE) [10]. A combination of ictal semiology, interictal and ictal EEG findings, MRI and PET/CT if applicable, was used to localize the seizure focus. All participants were right handedness. There was no significant difference in the subject age or gender among the three groups $(\mathrm{P}>0.05)$ (Table 1).

Table 1. Demographic and clinical variables.

\begin{tabular}{|c|c|c|c|}
\hline & LTLE & RTLE & $\mathrm{HC}$ \\
\hline Number & 42 & 42 & 41 \\
\hline Gender (female) & 15 & 18 & 25 \\
\hline Age (years) & $25.29 \pm 7.96$ & $26.96 \pm 8.33$ & $29.37 \pm 10.63$ \\
\hline duration (years) & $11.61 \pm 8.45$ & $11.22 \pm 8.69$ & - \\
\hline $\begin{array}{l}\text { Handedness } \\
\text { (right) }\end{array}$ & 42 & 42 & 41 \\
\hline $\begin{array}{c}\text { Seizure type } \\
\text { (FS/FS, sGTCS) } \\
\text { Seizure }\end{array}$ & $16 / 26$ & $16 / 26$ & - \\
\hline $\begin{array}{c}\text { frequency } \\
\text { (daily/weekly/mo } \\
\text { nthly/yearly) }\end{array}$ & $5 / 20 / 16 / 1$ & $5 / 15 / 18 / 4$ & - \\
\hline $\begin{array}{c}\text { History of } \\
\text { febrile } \\
\text { convulsions (yes) }\end{array}$ & 17 & 20 & - \\
\hline $\begin{array}{c}\text { MRI finding } \\
\text { (MTS/negative) }\end{array}$ & $29 / 13$ & $40 / 2$ & - \\
\hline $\begin{array}{l}\text { Number of } \\
\text { current AEDs } \\
(0 / 1 / 2 / 3 / 4)\end{array}$ & $\begin{array}{l}\text { 2/11/17/11/ } \\
1\end{array}$ & $1 / 17 / 17 / 5 / 2$ & - \\
\hline
\end{tabular}

Abbreviations: RTLE, Right TLE; LTLE, Left TLE; HC, Healthy Controls; FS, Focal Seizures; sGTCS, secondary Generalized TonicClonic Seizures; MTS, Mesial Temporal Sclerosis; AEDs, Antiepileptic drugs.

\section{B. Image acquisition}

High-resolution T1-weighted images were acquired using spoiled gradient recalled sequence on a $3 \mathrm{~T}$ MRI system (Tim Trio; Siemens, Erlangen, Germany) with an eight-channel head coil at West China Hospital. The main parameters included: repetition time $(\mathrm{TR})=1900 \mathrm{~ms}$; echo time $(\mathrm{TE})=2.26 \mathrm{~ms}$; flip angle $(\mathrm{FA})=9^{\circ}$, field of view $(\mathrm{FOV})=256 \times 256 \mathrm{~mm}^{2}$; voxel size $=1.0 \times 1.0 \times 1.0 \mathrm{~mm}^{3}, 176$ slices. T2-weighted images were acquired using a turbo spin echo (TSE) sequence (TR: $6100 \mathrm{~ms}$; TE: $97 \mathrm{~ms}$; FA: $120^{\circ}$; FOV: $230 \mathrm{~mm}$; voxel size $=0.6 \times 0.6 \times 3.9$ $\mathrm{mm}^{3} ; 35$ slices). This study was approved by the local ethics committee and informed consent was obtained from all subjects. Ten of the 84 patients have been previously reported in our prior article which explored the dynamic changes of white matter microstructure measured by diffusion tensor imaging in mesial temporal lobe epilepsy patients following anterior temporal lobectomy [11]. Different than the previous research, the current study investigated abnormalities of myelin in temporal lobe epilepsy by using T1- and T2-weighted MRI data.

\section{DATA PROCESSING}

A flowchart is provided in Fig. 1.

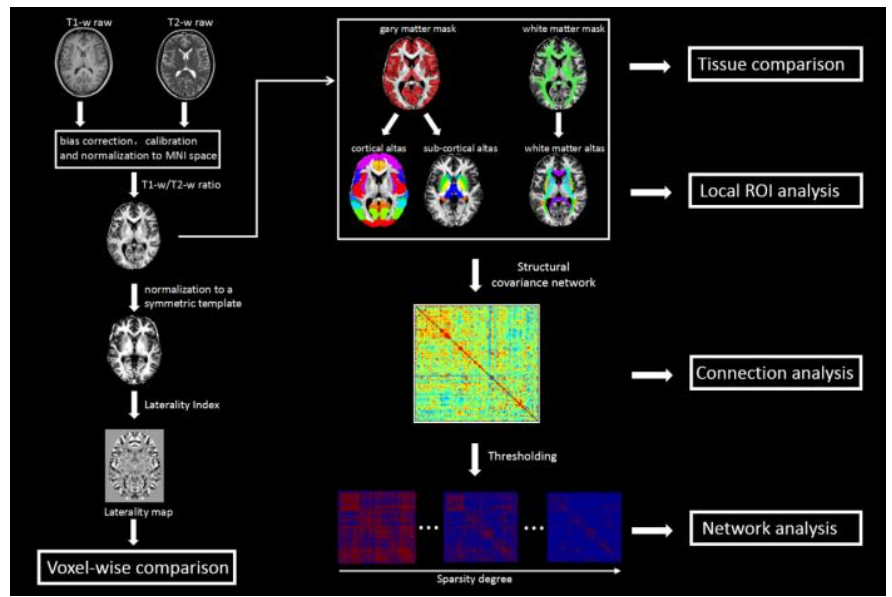

Fig. 1. The flowchart of data processing. First, the T1w and T2w images were pre-processed by the bias correction, intensity calibration and normalization using the MR Tool-Multimodal Mapping (http://www.bindgroup.eu/wp-content/uploads/2017/02/mrtool-

v1.3.1.zip) implemented in SPM12 (http://www.fil.ion.ucl.ac.uk/spm/software/spm12/) to obtain the T1w/T2w map. Second, the averaged T1w/T2w value was extracted from each ROI of JHU ICBM-DTI-81 white-matter atlas and the HarvardOxford grey-matter atlas. Third, the T1w/T2w structural covariance network (SCN) was built according to the strategy of the structural covariance network. The connection of the SCN was defined as the Pearson's correlation coefficient across subjects between each pair of ROls. Hence, the interregional correlation matrix $(\mathrm{N} \times \mathrm{N}, \mathrm{N}=160)$ was obtained to represent the similarity or synchronized co-variations in T1w/T2w. Next, we used graph theory to characterize the SCN attributes. Finally, the laterality analysis was performed by first co-registering the T1w/T2w map into a symmetric template. Subsequently, we calculated the laterality index (LI) for each homotopic voxel and thus obtained a laterality map for each subject.

\section{A. T1w/T2w mapping}

$\mathrm{T} 1 \mathrm{w}$ and $\mathrm{T} 2 \mathrm{w}$ images were pre-processed and combined, using a dedicated workflow outlined in previous studies [12]. This process included bias correction and intensity calibration 
on each of the T1w and T2 images and subsequent calculation of the ratio between the two images. In detail, each subject's $\mathrm{T} 2 \mathrm{w}$ image was co-registered to the T1w images using a rigidbody transformation. Second, bias correction was conducted on the $\mathrm{T} 1 \mathrm{w}$ and $\mathrm{T} 2 \mathrm{w}$ images separately. After correction for intensity non-uniformity, the $\mathrm{T} 1 \mathrm{w}$ and $\mathrm{T} 2 \mathrm{w}$ images were processed to standardize their intensity using a linear scaling procedure [12]. Subsequently, the ratio was calculated to obtain the $\mathrm{T} 1 \mathrm{w} / \mathrm{T} 2 \mathrm{w}$ image. Finally, the $\mathrm{T} 1 \mathrm{w} / \mathrm{T} 2 \mathrm{w}$ image was normalized to the Montreal Neurological Institute (MNI) space. The entire $\mathrm{T} 1 \mathrm{w} / \mathrm{T} 2 \mathrm{w}$ image processing was performed using the MR Tool-Multimodal Mapping (Release 1.3.1, http://www.bindgroup.eu/wp-content/uploads/2017/02/mrtoolv1.3.1.zip), a MATLAB-based toolkit requiring SPM version 12 (http://www.fil.ion.ucl.ac.uk/spm/software/spm12/).

\section{B. Tissue and ROI analysis}

The grey-matter (GM) and WM masks were defined based on the tissue probability map provided in SPM12 (threshold $=50 \%$ ). The average $\mathrm{T} 1 \mathrm{w} / \mathrm{T} 2 \mathrm{w}$ intensity within GM and WM masks was calculated. To gain further insight to the precise regions, the WM component was refined into 48 ROIs (21 bundles in each hemisphere and 6 commissure bundles) using the JHU ICBM-DTI-81 WM atlas [13], and the GM cortical (96 ROIs) and subcortical (16 ROIs) components were refined based on the Harvard-Oxford GM atlas [14]. The average $\mathrm{T} 1 \mathrm{w} / \mathrm{T} 2 \mathrm{w}$ intensity value was extracted from each ROI. ANCOVA was performed to examine the group difference among the LTLE, RTLE and HC, with age and gender as the covariates. The $P$ value $<0.05 / 160$ was considered significant for the multiple comparison correction (Bonferroni correction). In addition, the post hoc tests were performed for the comparisons between any two groups.

\section{T1w/T2w structural covariance network analysis}

The T1w/T2w structural covariance network (SCN) was built as described in the previous study by Melie-Garcia [15]. Based on JHU ICBM-DTI-81 WM atlas and the HarvardOxford GM atlas, the entire brain was parcellated into $48 \mathrm{WM}$ ROIs and 112 GM ROIs. The T1w/T2w data matrix was $\mathrm{M} \times$ $\mathrm{N}$, where $\mathrm{M}$ is the number of subjects and $\mathrm{N}$ represents the ROI number ( $\mathrm{N}=160$ in the current study). A linear regression was conducted on the $\mathrm{T} 1 \mathrm{w} / \mathrm{T} 2 \mathrm{w}$ data matrix to remove the effects of gender, age and age ${ }^{2}$ [15]. The connection of the SCN was defined as the Pearson's correlation coefficient across subjects between each pair of ROIs. Hence, the interregional correlation matrix $(\mathrm{N} \times \mathrm{N}, \mathrm{N}=160)$ was obtained to represent the similarity or synchronized co-variations in $\mathrm{T} 1 \mathrm{w} / \mathrm{T} 2 \mathrm{w}$. To avoid the influence of the weak correlations, one sample $t$ test was performed to determine the significant correlation $(\mathrm{P}<0.05$, FWE correction). To compare the group differences in the connection strength, a permutation test was performed [16]. In brief, we randomly assigned the group labels across subjects and re-generated the SCN, which was repeated 100,000 times. We computed the difference of each connection between the two random groups and generated a distribution of the null hypothesis of equality in connection strength between groups.
Based on the location of the real group difference of connection strength within the distribution of the null hypothesis, a $\mathrm{p}$ value was calculated for each connection.

Next, we used graph theory to characterize the SCN attributes. The sparse binary graphs were created using a range of sparsity degrees that varied from 0.1 to 0.4 with a step of 0.02 [15]. Subsequently, the network attributes, including clustering coefficient, characteristic path length, and global and local efficiency, were computed for the LTLE, RTLE and HC groups for each sparsity threshold. The formula and interpretations of graph measures can be found in prior research [17]. Finally, we calculated the area under the curve (AUC) for each network metric. The AUC metric can provide an integrated scalar for brain network topological characterization independent of a single threshold selection [18]. Statistical significance of the group differences was assessed using the permutation test, with a P-value < 0.05 [16]. In detail, group labels were randomly assigned across all subjects and the SCN attributes of each group was recalculated 10,000 times. The permutation processing allows for non-parametric estimation of the null distribution for the difference between any two groups.

\section{Laterality analysis}

Due to the differences of geometric configuration between cerebral hemispheres, each subject's $\mathrm{T} 1 \mathrm{w} / \mathrm{T} 2 \mathrm{w}$ images were nonlinearly registered into a symmetrical template [19]. Referring to previous studies [19], we created the symmetrical template using the following steps. First, all subjects' normalized $\mathrm{T} 1 \mathrm{w} / \mathrm{T} 2 \mathrm{w}$ images were averaged to obtain a group mean template. Then, the mean group template was left-to-right flipped and re-averaged to create a symmetrical template [19]. In the symmetrical brain space, we calculated the laterality index (LI) for each homotopic voxel [20], that is:

$\mathrm{LI}_{\mathrm{LR}}=\frac{\text { Intensity }_{\text {left }}-\text { Intensity }_{\text {right }}}{\text { Intensity }_{\text {left }}+\text { Intensity }_{\text {right }}}$

where Intensityleft represents the left $\mathrm{T} 1 \mathrm{w} / \mathrm{T} 2 \mathrm{w}$ intensity and Intensity ${ }_{\text {right }}$ represents the right $\mathrm{T} 1 \mathrm{w} / \mathrm{T} 2 \mathrm{w}$ intensity for each homotopic voxel.

By the LI calculation of each homotopic voxel, we obtained a laterality map for each subject. Voxel-wise ANCOVA was performed on the laterality maps to compare the differences among the LTLE, RTLE and HC, after controlling for the effects of gender and age. Multiple comparisons correction was performed using cluster-wise FDR correction (corrected $\mathrm{P}<0.05)$ [21].

\section{Results}

\section{A. Tissue and ROI analysis}

Consistent with a previous study [22], tissue comparison confirmed that WM had higher T1w/T2w intensity than GM (Fig. 2A). In addition, we found $\mathrm{T} 1 \mathrm{w} / \mathrm{T} 2 \mathrm{w}$ reductions in TLE for GM and WM, compared to controls (Fig. 2A).

We then focused our investigation on specific brain regions within the WM and GM including sub-cortical and cortical regions. GM sub-cortical ROI comparisons showed that, 
compared with HC, both LTLE and RTLE groups had decreased $\mathrm{T} 1 \mathrm{w} / \mathrm{T} 2 \mathrm{w}$ intensity in the left amygdala, left thalamus and bilateral hippocampus (Fig. S1, Table S1), with more severe reduction in the ipsilateral hippocampus (Fig. 2B and Fig. 2C) in each patient group. WM ROI analysis indicated lower $\mathrm{T} 1 \mathrm{w} / \mathrm{T} 2 \mathrm{w}$ in bilateral cingulum adjoining the hippocampus (CGH) and fornix body (FIX) in both TLE groups (Fig. S2, Table S2). In left CGH, the LTLE exhibited reduced T1w/T2w intensity compared to the RTLE (Fig. 2D). Cortical ROI analysis found that compared with HC, both TLE groups had lower $\mathrm{T} 1 \mathrm{w} / \mathrm{T} 2 \mathrm{w}$ in the temporal lobes (bilateral temporal pole [TP], left anterior superior temporal gyrus [ASTG], right posterior temporal fusiform cortex [PTFC], left posterior middle temporal gyrus [PMTG], left anterior inferior temporal gyrus [AITG], left posterior parahippocampal gyrus [PPHIP]), frontal lobes (left pars triangularis of inferior frontal gyrus [TriIFG], bilateral middle frontal gyrus [MFG], left subcallosal cortex [SCC], and left frontal pole [FP]), occipital lobes (left superior lateral occipital cortex [SLOC] and right occipital pole $[\mathrm{OP}]$ ), parietal (left anterior supramarginal gyrus [ASMG]) and insular lobes (right insula [INS]) (Fig. S3, Table S3). Additionally, the LTLE showed more severe decreases in the left temporal pole (TP) (Fig. 2E), left SCC (Fig. 2F) and left PPHIP (Fig. 2G), compared with RTLE.
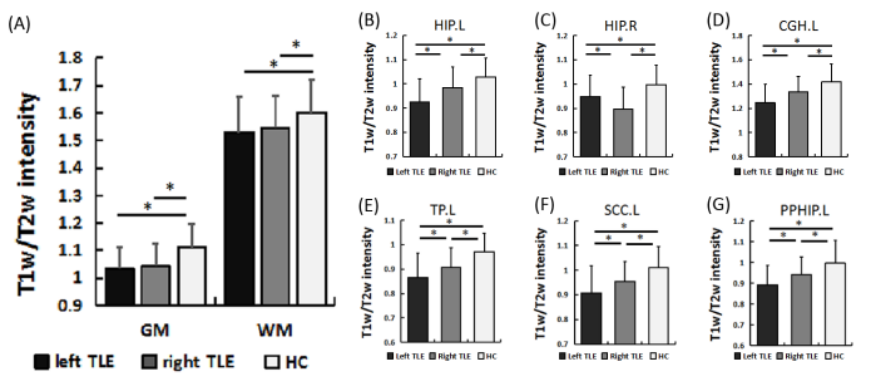

Fig. 2. Differences of $T 1 w / T 2 w$ intensity among the left TLE, right TLE and $\mathrm{HC}$ in (A) GM and WM masks, (B) left HIP, (C) right HIP, (D) left CGH, (E) left TP, (F) left SCC and (G) left PPHIP. The $P<0.05 / 160$ was considered significant for the multiple comparisons correction (Bonferroni correction). The error bars represent standard deviations. TLE, temporal lobe epilepsy; HC, healthy controls; GM, grey matter; WM, white matter; HIP, hippocampus; $\mathrm{CGH}$, cingulum adjoining the hippocampus; TP, temporal pole; SCC, subcallosal cortex; PPHIP, posterior parahippocampal gyrus. * represents $\mathrm{P}<0.05$, Bonferroni correction.

\section{B. SCN analysis}

The edges of the SCN indicated significant correlations between $\mathrm{T} 1 \mathrm{w} / \mathrm{T} 2 \mathrm{w}$ measures of specific ROIs across subjects. By comparing patients and healthy controls, the edges can be described as decreased connections (ROI-ROI correlation was significantly lower in patients compared with controls) and increased connections (ROI-ROI correlation was significantly higher in patients compared with controls) by the permutation test (Fig. 3A). In total, compared with HC, the LTLE group showed more changed connections than RTLE (59 vs. 35) (Table 2). Furthermore, there were more increased connections than decreased connections (29 vs. 19) in the LTLE group, while there were more decreased connections than increased connections (20 vs. 5) in the RTLE group (Table 2). In both LTLE and RTLE groups, there were more extensive changes in the cortical ROI to cortical ROI connection pattern, compared with other connection patterns (Table 2), such as subcortical ROI to subcortical ROI connection or WM ROI to WM ROI connection. In addition, there were more increased cortical-tocortical connections in LTLE than RTLE (29 vs. 5), especially for the frontotemporal connectivity.

Table 2. Decreased connections and increased connections of T1w/T2w structural covariance network in left TLE and right TLE.

\begin{tabular}{ccccc}
\hline Connection & \multicolumn{2}{c}{ Decrease } & \multicolumn{2}{c}{ Increase } \\
\cline { 2 - 5 } & LTLE & RTLE & LTLE & RTLE \\
\hline Cortical ROI to cortical ROI & 19 & 20 & 29 & 5 \\
$\begin{array}{c}\text { connection } \\
\text { Subcortical ROI to }\end{array}$ & 1 & 0 & 3 & 2 \\
$\begin{array}{c}\text { Subcortical ROI connection } \\
\text { WM ROI to WM ROI }\end{array}$ & 2 & 3 & 0 & 2 \\
$\quad \begin{array}{c}\text { connection } \\
\text { Cortical ROI to subcortical } \\
\quad \text { ROI connection }\end{array}$ & 2 & 1 & 0 & 2 \\
$\begin{array}{c}\text { Cortical ROI to WM ROI } \\
\text { connection }\end{array}$ & 3 & 0 & 0 & 0 \\
$\begin{array}{c}\text { Subcortical ROI to WM ROI } \\
\text { connection } \\
\text { Total }\end{array}$ & 0 & 0 & 2 & 0 \\
\hline
\end{tabular}

(A)
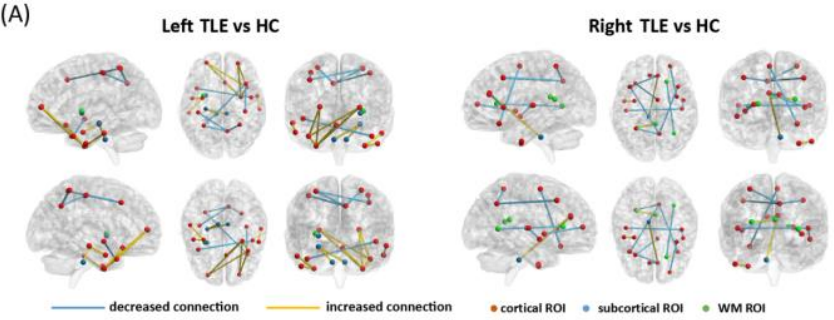

(B)
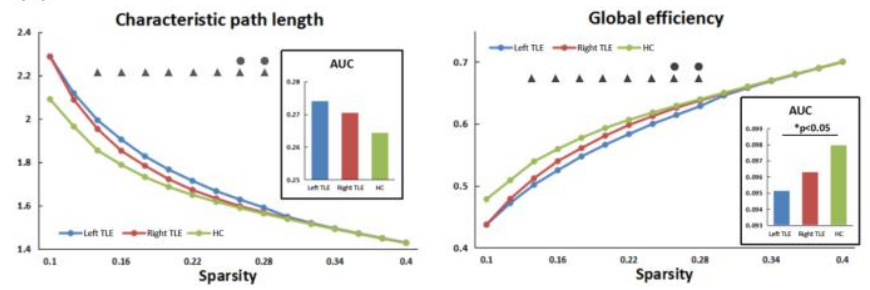

Fig. 3. Changes of the $T 1 w / T 2 w$ structural covariance network in left TLE and right TLE. (A) By comparing patients and healthy controls, the edges can be described as decreased connections (ROI-ROI correlation was significantly lower in patients compared with controls) and increased connections (ROI-ROI correlation was significantly higher in patients compared with controls). (B) Differences of attributes in the structural covariance network among the left TLE, right TLE and HC. The triangles represent significant differences between left TLE and HC $(P<0.05)$. The circles represent significant differences between left TLE and right TLE $(P<0.05)$. TLE, temporal lobe epilepsy; $H C$, healthy controls.

The permutation test revealed that compared with $\mathrm{HC}$, only the LTLE group had higher characteristic path length and lower global efficiency for a range of sparsity threshold from 0.14 to 0.28 (All Ps<0.05) (Fig. 3B). Moreover, the comparisons between two patient groups showed higher characteristic path length and lower global efficiency in LTLE at the sparsity of 0.26 and $0.28(\mathrm{P}<0.05)$ (Fig. 3B). In addition, the AUC analysis showed that only the LTLE group exhibited a significant decrease $(\mathrm{P}<0.05)$ in the AUC of global efficiency and a marginally significant increase $(\mathrm{P}=0.050)$ in the AUC of characteristic path length, compared with HC (Fig. 3B). There was no significant difference among the healthy controls, LTLE 
and RTLE group, in terms of clustering coefficient and local efficiency (All Ps > 0.05).

\section{Laterality analysis}

The ANCOVA on the $\mathrm{LI}_{\mathrm{LR}}$ maps showed significant differences among the LTLE, RTLE and HC groups in anterior and posterior putamen, hippocampus and anterior temporal area ( $\mathrm{P}<0.05$, FDR correction) (Fig. 4A). The post hoc tests revealed that there was decreased $\mathrm{LI}_{\mathrm{LR}}$ in $\mathrm{LTLE}$ and increased $\mathrm{LI}_{\mathrm{LR}}$ in RTLE compared with $\mathrm{HC}$ (Ps $<0.05$, Bonferroni correction) (Fig. 4B). In detail, one-sample t-tests showed that the laterality of the anterior temporal area was significantly different from zero in all three groups (corrected Ps $<0.05$ ). The laterality of the hippocampus and posterior putamen existed in LTLE and RTLE (corrected Ps $<0.05$ ) but not in the HC. The laterality of the anterior putamen existed only in the RMTL (corrected $\mathrm{P}<0.05$ ). Due to the $\mathrm{LI}_{\mathrm{LR}}$ calculation (left-right), both LTLE and RTLE exhibited a consistent decrease of $\mathrm{T} 1 \mathrm{w} / \mathrm{T} 2 \mathrm{w} \mathrm{LI}_{\mathrm{LR}}$ at the ipsilateral hippocampus. Except for the hippocampus and anterior temporal area, both TLE groups showed a T1w/T2w $\mathrm{LI}_{\mathrm{LR}}$ decrease at the contralateral putamen (all Ps $<0.05$ ) (Fig. 4B).
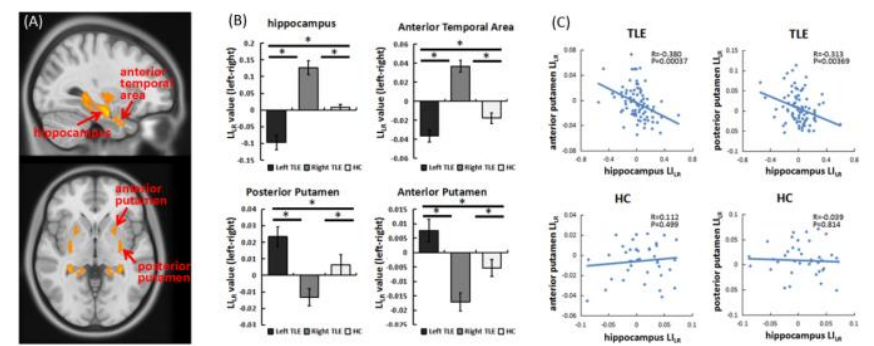

Fig. 4. Results of laterality analysis in TLE. (A) Brain regions with significant differences of $L I_{L R}$ maps among the left TLE, right TLE and $\mathrm{HC}$ groups by ANCOVA ( $\mathrm{P}<0.05$, FDR correction). (B) Altered $L_{L R}$ in the hippocampus and putamen in left TLE and right TLE compared with $\mathrm{HC}$ by post hoc analysis. (C) A significant correlation between hippocampal $\mathrm{LI}_{\mathrm{LR}}$ and anterior and posterior putamen's $\mathrm{LI}_{\mathrm{LR}}$ in a cohort of all TLE patients but not in HC. TLE, temporal lobe epilepsy; HC, healthy controls; $\mathrm{LI}$, laterality index. * represents $\mathrm{P}<0.05$, Bonferroni correction.

As there are structural connections between the hippocampus and putamen, we further investigated whether a damaging synchronization between the hippocampus and putamen exists. We found a significant Pearson's correlation between hippocampal $\mathrm{LI}_{\mathrm{LR}}$ and anterior and posterior putamen $\mathrm{LI}_{\mathrm{LR}}$ in a cohort of all TLE patients (anterior putamen: $\mathrm{R}=-0.380$, $\mathrm{P}<0.001$; anterior putamen: $\mathrm{R}=-0.313, \mathrm{P}=0.004)$ but not in the $\mathrm{HC}$ (anterior putamen: $\mathrm{R}=0.112, \mathrm{P}=0.499$; anterior putamen: $\mathrm{R}=-0.039, \mathrm{P}=0.814)$ (Fig. 4C).

In addition, we observed different alterations between putamen anterior and posterior regions in LTLE and RTLE. To better clarify the laterality changes in the putamen and perform a directed comparison between LTLE and RTLE, we redefined the laterality measure as the following equation:

$$
\mathrm{LI}_{\mathrm{IC}}=\frac{\text { Intensity }_{\mathrm{ips}}-\text { Intensity }_{\mathrm{con}}}{\text { Intensity }_{\mathrm{ips}}+\text { Intensity }_{\mathrm{con}}}
$$

where Intensity ${ }_{i p s}$ represents the ipsilateral $\mathrm{T} 1 \mathrm{w} / \mathrm{T} 2 \mathrm{w}$ intensity and Intensitycon represents the contralateral $\mathrm{T} 1 \mathrm{w} / \mathrm{T} 2 \mathrm{w}$ intensity for anterior and posterior putamen. A high value of the $\mathrm{LI}_{\mathrm{IC}}$ represented a high $\mathrm{T} 1 \mathrm{w} / \mathrm{T} 2 \mathrm{w}$ intensity on the contralateral putamen. We identified a significant interaction effect $(\mathrm{F}=4.26$, $\mathrm{P}<0.05$ ) between the side of epileptic focus (left or right) and the $\mathrm{LI}_{\mathrm{IC}}$ of the putamen anterior or posterior (Fig. S4) using the repeated measured ANOVA. Specifically, the LTLE showed a higher laterality at the posterior putamen, while the RTLE exhibited a higher laterality at the anterior putamen (Fig. S4).

To further investigate the ATL influence on the putamen, we focused on the following questions: Are there longitudinal changes in the $\mathrm{LI}_{\mathrm{IC}}$ of putamen after ATL? If so, how does the $\mathrm{T} 1 \mathrm{w} / \mathrm{T} 2 \mathrm{w}$ of bilateral putamen change? We measured the longitudinal changes in another longitudinal cohort of 14 refractory TLE patients who have both pre-ATL and post-ATL MRI data (see Supplementary Table S4 for details). Paired ttests indicated increased $\mathrm{LI}_{\mathrm{IC}}$ in both anterior $(\mathrm{T}=3.20, \mathrm{P}=0.007)$ and posterior putamen $(\mathrm{T}=8.47, \mathrm{P}<0.001)$ in the TLE patients after ATL (Fig. S5). Subsequently, to further determine which putamen subregions $\mathrm{T} 1 \mathrm{w} / \mathrm{T} 2 \mathrm{w}$ changes contribute to the increased $\mathrm{LI}_{\mathrm{IC}}$, we compared the longitudinal $\mathrm{T} 1 \mathrm{w} / \mathrm{T} 2 \mathrm{w}$ intensity alterations between pre-ATL and post-ATL in ipsilateral and contralateral putamen subregions. We found increased $\mathrm{T} 1 \mathrm{w} / \mathrm{T} 2 \mathrm{w}$ intensity only in the ipsilateral putamen after ATL (ipsilateral anterior putamen: $\mathrm{T}=2.91, \mathrm{P}=0.012$; ipsilateral posterior putamen: $\mathrm{T}=5.92, \mathrm{P}<0.001)($ Fig. $\mathbf{S 6}$ ).

\section{DISCUSSION}

This study used the $\mathrm{T} 1 \mathrm{w} / \mathrm{T} 2 \mathrm{w}$ ratio to measure in vivo the brain microstructural changes in a large cohort of TLE patients, which allowed separate baseline analysis of LTLE and RTLE. Another cohort of TLE patients undergoing ATL surgery enables a further validation study of longitudinal changes induced by the ATL. First, compared to the HC, both LTLE and RTLE groups showed decreased $\mathrm{T} 1 \mathrm{w} / \mathrm{T} 2 \mathrm{w}$ in cortical areas (mainly in frontotemporal regions), subcortical regions (hippocampus, amygdala and thalamus) and WM (CGH and FIX). Moreover, LTLE presented with lower T1w/T2w in left medial temporal region, compared to RTLE. Second, the SCN analysis indicated that compared to HC, both LTLE and RTLE groups had extensive changes of cortex-cortex connections. However, LTLE presented with more increased connections than RTLE. In addition, only the LTLE group exhibited a significant decrease in the global efficiency of SCN compared with HC. Third, the two TLE groups showed altered laterality of T1w/T2w in the hippocampus and putamen. Moreover, the $\mathrm{T} 1 \mathrm{w} / \mathrm{T} 2 \mathrm{w}$ at the two regions exhibited a negative correlation in the patient cohort. Additionally, we found that the LTLE showed a higher laterality at the posterior putamen, where the RTLE exhibited a higher laterality at the anterior putamen. Lastly, we found increased $\mathrm{T} 1 \mathrm{w} / \mathrm{T} 2 \mathrm{w}$ only in the ipsilateral putamen following the ATL. 


\section{A. T1w/T2w intensity decreases in cortical, subcortical regions and WM in TLE}

Across the two groups of TLE and HC, the comparisons using WM and GM as ROIs suggested a global reduction of T1w/T2w intensity in TLE. This result is consistent with current studies suggesting that TLE is a system-level disorder, with both GM and WM disruptions as the foundation for the clinical outcome of the pathology [23]. Regarding structural impairments in GM cortices, we found prominent alterations in TLE patients to be localized in the frontal and temporal lobes, which were consistent with previous studies revealing that abnormalities of frontal-temporal networks may be linked to cognitive impairments and pathological outcomes in TLE [24]. Regarding subcortical structures, we found $\mathrm{T} 1 \mathrm{w} / \mathrm{T} 2 \mathrm{w}$ decreases in the hippocampus, amygdala and thalamus. The results were consistent with cumulative evidence indicating a key role of a thalamotemporal network in the pathologic alterations of TLE [25]. Regarding the WM results, the current study identified a reduction of T1w/T2w intensity in the WM tracts of CGH and FIX in the TLE patients. Several studies have also reported consistent WM aberrations in hippocampal WM pathways [26]. Previous studies using diffusion tensor imaging have observed widespread microstructural organizational changes, such as reduced fractional anisotropy (FA), which is possibly linked to myelination alterations [27]. Previous work has reported aberrant myelin content in children with epilepsy [28]. Moreover, a recent review also suggested a relationship between pathophysiology of epilepsy and myelin abnormalities [29]. The present findings provided brain microstructural changes of T1w/T2 $w$ ratios in TLE, using a combination of two MRI measures [12]. The observed changes in $\mathrm{T} 1 \mathrm{w} / \mathrm{T} 2 \mathrm{w}$ used in this study could be due to changes in myelination of TLE patients, but it should be recognized that other microstructural changes may be responsible.

\section{B. T1w/T2w structural covariance network is distinct in} left and right TLE

The structural covariance network method has been widely used to investigate the concurrent changes of brain structures (i.e., cortical thickness) in normal and pathological brain [30]. To characterize the concurrent changes in $\mathrm{T} 1 \mathrm{w} / \mathrm{T} 2 \mathrm{w}$ across brain anatomical structures in vivo, this study built a $\mathrm{SCN}$ by calculating the synchronicity of the variations in $\mathrm{T} 1 \mathrm{w} / \mathrm{T} 2 \mathrm{w}$ between anatomical regions. The $\mathrm{T} 1 \mathrm{w} / \mathrm{T} 2 \mathrm{w}$ covariance network analysis pointed to more severe and more extensive changes in LTLE than RTLE. Such distinct network alterations agree with several structural studies of GM and WM [31, 32]. For example, morphological analysis on GM volume showed a stronger reduction in patients with left-sided seizure origin [33]. Investigations of FA in WM tracts identified more widespread deficits in LTLE [34]. Focke et al. also found that the WM tract of arcuate fasciculus was more affected in LTLE [35]. Wang and colleagues reported aberrant topological patterns of metabolic covariance networks in TLE [36]. In addition, Campos et al. indicated that LTLE had a more intricate bilateral bi-hemispheric dysfunction compared to RTLE using restingstate fMRI [9]. In total, our findings identified a distinct pathological network of $\mathrm{T} 1 \mathrm{w} / \mathrm{T} 2 \mathrm{w}$, which exhibited more connectome alterations in LTLE than RTLE, supporting previous suggestions that LTLE is a more severe network disease than RTLE [31].

The topological properties of the structural covariance network were also altered in TLE. The "characteristic path length" was statistically higher in LTLE. The increased "characteristic path length" was accompanied by a decreased "global efficiency" in principle. According to the graphical theory, higher "characteristic path length" represents higher "wiring cost" for information communication within the network. Additionally, human brain studies also demonstrated that the interpretation is reasonable. For example, Melie-Garcia and colleagues reported an increasing characteristic path length in an old-age group compared to young-age subjects, suggesting that worse network efficiency is related to poor performances in older subjects [15]. The altered topological organization in TLE patients indicated worse network efficiency, suggesting insufficient information interactions in the brain. These findings provided further support for the hypothesis that abnormalities of brain network efficiency in LTLE may be more serious than in RTLE [37].

\section{Laterality of T1w/T2w analysis: the putamen may play a crucial role in controlling epileptic seizures}

Asymmetry is used for pathological detection for several reasons. First, the degree of asymmetry is commonly reviewed by expert radiologists who visually inspect the neuroimaging data in search of pathology. Second, asymmetry measurement can reduce confounding factors from differences in diseases, scanner parameters and neuroimaging modalities, by using each subject as a control for itself. Third, previous studies have shown that asymmetric measures are more sensitive to pathological characterization than raw voxel values [38].

In TLE, previous studies have shown asymmetric atrophy in mesial temporal structures and asymmetric reduction of glucose metabolism in the temporal lobes [38]. A recent study found that TLE patients showed greater grey matter volume in contralateral hippocampus and subfields, indicating volumetric asymmetry in TLE [39]. Resting-state fMRI studies also reported decreased ipsilateral functional connectivity within the mesial temporal lobes in TLE [40]. Consistent with these findings, we observed asymmetric anomalies related to pathological changes in the hippocampus, using the ratio of T1w/T2w images. In addition, the current study found asymmetric alteration of $\mathrm{T} 1 \mathrm{w} / \mathrm{T} 2 \mathrm{w}$ in the putamen in TLE. The putamen, as a subcortical region, is a principal anatomical component of the basal ganglia, which receives inputs from the cortical areas and sends information back to the cortex via the thalamus [41]. The basal ganglia shows intimate structural connections with the brain limbic system, especially the hippocampus [42]. Reduction of grey-matter volume has also been reported in the basal ganglia, including the putamen in TLE patients [43]. Additionally, aberrant functional connectivity in the basal ganglia and hippocampus has been noted previously in TLE [44]. Furthermore, we found that the $\mathrm{T} 1 \mathrm{w} / \mathrm{T} 2 \mathrm{w}$ values of the putamen negatively correlated with that 
of the hippocampus. According to the structural connection and functional association between the hippocampus and putamen, we hypothesized that the putamen may play a transfer station role in damage spreading induced by epileptic seizures from the hippocampus. The hypothesis was further supported by the finding that the $\mathrm{T} 1 \mathrm{w} / \mathrm{T} 2 \mathrm{w}$ of the putamen increased after the ATL. These results are consistent with previous studies reporting that the basal ganglia plays an important role in control of the epileptic activity through the brain [45]. However, we found the myelination improvement only appeared in the ipsilateral putamen, which may suggest a lateralization effect of the putamen improvement induced by the ATL. These findings may guide the development of new intervention targets or treatment strategies that may modulate morphological reorganization in specific subcortical nuclei.

In addition, we found different patterns of $\mathrm{T} 1 \mathrm{w} / \mathrm{T} 2 \mathrm{w}$ laterality in putamen subregions between LTLE and RTLE, revealed by a higher laterality at the posterior putamen in the LTLE, but a higher laterality at the anterior putamen in the RTLE. This finding provided evidence for identifiable differences of myelination damages in the putamen between LTLE and RTLE.

\section{Limitations}

The current study had several limitations. First, the present study used the $\mathrm{T} 1 \mathrm{w} / \mathrm{T} 2 \mathrm{w}$ ratio method to characterize the brain changes of myelination. It is necessary to investigate the reproducibility using other measures of myelination mapping, such as magnetization transfer ratio (MTR), although previous studies have demonstrated a high degree of correlation between MTR and T1w/T2w ratio [46]. Second, myelination largely contributes to the ratio of the T1w/T2 $\mathrm{w}$ images; however, some factors, including inflammation, oedema, metabolism, atrophy or iron accumulation, may also contribute to the $\mathrm{T} 1 \mathrm{w} / \mathrm{T} 2 \mathrm{w}$ signals [12]. The $\mathrm{T} 1 \mathrm{w} / \mathrm{T} 2 \mathrm{w}$ ratio used in the current study is an estimate of $\mathrm{T} 1 \mathrm{w} / \mathrm{T} 2 \mathrm{w}$, not a fully quantitative measure of it (i.e., quantitative $\mathrm{T} 1$ and $\mathrm{T} 2$ relaxometry was not performed). Third, the cohorts of patients before and after ATL surgery enable a baseline comparison and a further investigation of longitudinal changes following surgery. Not all patients underwent the ATL surgery. In addition, the dynamic brain changes should be further investigated on data from multiple postoperative time points. Finally, disease duration and medication use may also affect the secondary changes in the T1/T2 ratio map. A larger sample size with greater statistical power is needed to further verify the significance of the group differences.

\section{CONCLUSION}

This study demonstrated $\mathrm{T} 1 \mathrm{w} / \mathrm{T} 2 \mathrm{w}$ reductions in the frontotemporal and thalamic regions and extensive disconnections of a $\mathrm{T} 1 \mathrm{w} / \mathrm{T} 2 \mathrm{w}$ structural covariance network, providing evidence that TLE is a system disorder with widespread disruptions at regional and network levels. The $\mathrm{T} 1 \mathrm{w} / \mathrm{T} 2 \mathrm{w}$ laterality of the putamen at baseline was altered and negatively related with the hippocampus in TLE. Furthermore, ATL surgery increased $\mathrm{T} 1 \mathrm{w} / \mathrm{T} 2 \mathrm{w}$ values in the ipsilateral putamen. This finding suggested that the putamen may play a transfer station role in damage spreading induced by epileptic seizures from the hippocampus. Last, we found identifiable differences of T1w/T2w changes between LTLE and RTLE, revealed by more increased connections and decreased global efficiency of a covariance network in LTLE.

\section{REFERENCES}

[1] S. Wiebe, and N. Jette, "Pharmacoresistance and the role of surgery in difficult to treat epilepsy," Nat Rev Neurol, vol. 8, no. 12, pp. 66977, Dec, 2012

[2] L. Caciagli, A. Bernasconi, S. Wiebe et al., "A meta-analysis on progressive atrophy in intractable temporal lobe epilepsy: Time is brain?," Neurology, vol. 89, no. 5, pp. 506-516, Aug 1, 2017.

[3] M. Thom, J. L. Holton, C. D'Arrigo et al., "Microdysgenesis with abnormal cortical myelinated fibres in temporal lobe epilepsy: a histopathological study with calbindin D-28-K immunohistochemistry," Neuropathol Appl Neurobiol, vol. 26, no. 3, pp. 251-7, Jun, 2000.

[4] R. Garbelli, G. Milesi, V. Medici et al., "Blurring in patients with temporal lobe epilepsy: clinical, high-field imaging and ultrastructural study," Brain, vol. 135, no. Pt 8, pp. 2337-49, Aug, 2012.

[5] M. F. Glasser, and D. C. Van Essen, "Mapping human cortical areas in vivo based on myelin content as revealed by $\mathrm{T} 1$ - and T2-weighted MRI," J Neurosci, vol. 31, no. 32, pp. 11597-616, Aug 10, 2011.

[6] L. L. Chao, D. Tosun, S. H. Woodward et al., "Preliminary Evidence of Increased Hippocampal Myelin Content in Veterans with Posttraumatic Stress Disorder," Front Behav Neurosci, vol. 9, pp. $333,2015$.

[7] T. Granberg, Q. Fan, C. A. Treaba et al., "In vivo characterization of cortical and white matter neuroaxonal pathology in early multiple sclerosis," Brain, vol. 140, no. 11, pp. 2912-2926, Nov 1, 2017.

[8] M. Liu, B. C. Bernhardt, A. Bernasconi et al., "Gray matter structural compromise is equally distributed in left and right temporal lobe epilepsy," Hum Brain Mapp, vol. 37, no. 2, pp. 51524, Feb, 2016.

[9] B. M. de Campos, A. C. Coan, C. Lin Yasuda et al., "Large-scale brain networks are distinctly affected in right and left mesial temporal lobe epilepsy," Hum Brain Mapp, vol. 37, no. 9, pp. 313752, Sep, 2016

[10] R. S. Fisher, J. H. Cross, J. A. French et al., "Operational classification of seizure types by the International League Against Epilepsy: Position Paper of the ILAE Commission for Classification and Terminology," Epilepsia, vol. 58, no. 4, pp. 522-530, Apr, 2017.

[11] W. Li, D. An, X. Tong et al., "Different patterns of white matter changes after successful surgery of mesial temporal lobe epilepsy," Neuroimage Clin, vol. 21, pp. 101631, 2019.

[12] M. Ganzetti, N. Wenderoth, and D. Mantini, "Whole brain myelin mapping using T1- and T2-weighted MR imaging data," Front Hum Neurosci, vol. 8, pp. 671, 2014.

[13] S. Mori, K. Oishi, H. Jiang et al., "Stereotaxic white matter atlas based on diffusion tensor imaging in an ICBM template," Neuroimage, vol. 40, no. 2, pp. 570-582, Apr 1, 2008.

[14] R. S. Desikan, F. Segonne, B. Fischl et al., "An automated labeling system for subdividing the human cerebral cortex on MRI scans into gyral based regions of interest," Neuroimage, vol. 31, no. 3, pp. 96880, Jul 1, 2006.

[15] L. Melie-Garcia, D. Slater, A. Ruef et al., "Networks of myelin covariance," Hum Brain Mapp, vol. 39, no. 4, pp. 1532-1554, Apr, 2018.

[16] L. Palaniyappan, B. Park, V. Balain et al., "Abnormalities in structural covariance of cortical gyrification in schizophrenia," Brain Struct Funct, vol. 220, no. 4, pp. 2059-71, Jul, 2015.

[17] M. Rubinov, and O. Sporns, "Complex network measures of brain connectivity: uses and interpretations," Neuroimage, vol. 52, no. 3, pp. 1059-69, Sep, 2010.

[18] D. Lei, K. Li, L. Li et al., "Disrupted Functional Brain Connectome in Patients with Posttraumatic Stress Disorder," Radiology, vol. 276, no. 3, pp. 818-27, Sep, 2015.

[19] X. N. Zuo, C. Kelly, A. Di Martino et al., "Growing together and growing apart: regional and sex differences in the lifespan 
[20] O. Agcaoglu, R. Miller, A. R. Mayer et al., "Lateralization of resting state networks and relationship to age and gender," Neuroimage, vol. 104, pp. 310-25, Jan 1, 2015.

[21] J. R. Chumbley, and K. J. Friston, "False discovery rate revisited: FDR and topological inference using Gaussian random fields," Neuroimage, vol. 44, no. 1, pp. 62-70, Jan 1, 2009.

[22] M. Ganzetti, N. Wenderoth, and D. Mantini, "Mapping pathological changes in brain structure by combining T1- and T2-weighted MR imaging data," Neuroradiology, vol. 57, no. 9, pp. 917-28, Sep, 2015.

[23] B. C. Bernhardt, S. Hong, A. Bernasconi et al., "Imaging structural and functional brain networks in temporal lobe epilepsy," Front Hum Neurosci, vol. 7, pp. 624, Oct 1, 2013.

[24] G. Hwang, K. Dabbs, L. Conant et al., "Cognitive slowing and its underlying neurobiology in temporal lobe epilepsy," Cortex, vol. 117 , pp. 41-52, Mar 5, 2019.

[25] M. Guye, J. Regis, M. Tamura et al., "The role of corticothalamic coupling in human temporal lobe epilepsy," Brain, vol. 129, no. Pt 7, pp. 1917-28, Jul, 2006.

[26] G. P. Winston, J. Stretton, M. K. Sidhu et al., "Progressive white matter changes following anterior temporal lobe resection for epilepsy," Neuroimage Clin, vol. 4, pp. 190-200, 2014.

[27] A. Reyes, E. Kaestner, N. Bahrami et al., "Cognitive phenotypes in temporal lobe epilepsy are associated with distinct patterns of white matter network abnormalities," Neurology, vol. 92, no. 17, pp. e1957-e1968, Apr 23, 2019.

[28] G. S. Drenthen, E. L. A. Fonseca Wald, W. H. Backes et al., "Lower myelin-water content of the frontal lobe in childhood absence epilepsy," Epilepsia, vol. 60, no. 8, pp. 1689-1696, Aug, 2019.

[29] G. S. Drenthen, W. H. Backes, A. P. Aldenkamp et al., "On the merits of non-invasive myelin imaging in epilepsy, a literature review," J Neurosci Methods, vol. 338, pp. 108687, May 15, 2020.

[30] A. Alexander-Bloch, J. N. Giedd, and E. Bullmore, "Imaging structural co-variance between human brain regions," Nat Rev Neurosci, vol. 14, no. 5, pp. 322-36, May, 2013.

[31] P. Besson, V. Dinkelacker, R. Valabregue et al., "Structural connectivity differences in left and right temporal lobe epilepsy," Neuroimage, vol. 100, pp. 135-44, Oct 15, 2014

[32] S. S. Keller, U. C. Wieshmann, C. E. Mackay et al., "Voxel based morphometry of grey matter abnormalities in patients with medically intractable temporal lobe epilepsy: effects of side of seizure onset and epilepsy duration," $J$ Neurol Neurosurg Psychiatry, vol. 73, no. 6, pp. 648-55, Dec, 2002.

[33] L. Bonilha, A. Alessio, C. Rorden et al., "Extrahippocampal gray matter atrophy and memory impairment in patients with medial temporal lobe epilepsy," Hum Brain Mapp, vol. 28, no. 12, pp. 1376-90, Dec, 2007.

[34] D. Pustina, G. Doucet, M. Sperling et al., "Increased microstructural white matter correlations in left, but not right, temporal lobe epilepsy," Hum Brain Mapp, vol. 36, no. 1, pp. 85-98, Jan, 2015.

[35] N. K. Focke, M. Yogarajah, S. B. Bonelli et al., "Voxel-based diffusion tensor imaging in patients with mesial temporal lobe epilepsy and hippocampal sclerosis," Neuroimage, vol. 40, no. 2, pp. 728-737, Apr 1, 2008.

[36] K. L. Wang, W. Hu, T. H. Liu et al., "Metabolic covariance networks combining graph theory measuring aberrant topological patterns in mesial temporal lobe epilepsy," CNS Neurosci Ther, vol. 25, no. 3, pp. 396-408, Mar, 2019.

[37] N. Kemmotsu, H. M. Girard, B. C. Bernhardt et al., "MRI analysis in temporal lobe epilepsy: cortical thinning and white matter disruptions are related to side of seizure onset," Epilepsia, vol. 52, no. 12, pp. 2257-66, Dec, 2011.

[38] D. Pustina, B. Avants, M. Sperling et al., "Predicting the laterality of temporal lobe epilepsy from PET, MRI, and DTI: A multimodal study," Neuroimage Clin, vol. 9, pp. 20-31, 2015.

[39] P. Shah, D. S. Bassett, L. E. M. Wisse et al., "Structural and functional asymmetry of medial temporal subregions in unilateral temporal lobe epilepsy: A 7T MRI study," Hum Brain Mapp, vol. 40, no. 8, pp. 2390-2398, Jun 1, 2019.

[40] G. Bettus, F. Bartolomei, S. Confort-Gouny et al., "Role of resting state functional connectivity MRI in presurgical investigation of mesial temporal lobe epilepsy," J Neurol Neurosurg Psychiatry, vol. 81, no. 10, pp. 1147-54, Oct, 2010.
[41] M. Duan, X. Chen, H. He et al., "Altered Basal Ganglia Network Integration in Schizophrenia," Front Hum Neurosci, vol. 9, pp. 561, 2015.

[42] B. H. Bland, "The power of theta: providing insights into the role of the hippocampal formation in sensorimotor integration," Hippocampus, vol. 14, no. 5, pp. 537-8, 2004.

[43] J. S. Kim, D. L. Koo, E. Y. Joo et al., "Asymmetric Gray Matter Volume Changes Associated with Epilepsy Duration and Seizure Frequency in Temporal-Lobe-Epilepsy Patients with Favorable Surgical Outcome," J Clin Neurol, vol. 12, no. 3, pp. 323-31, Jul, 2016.

[44] Z. Haneef, A. Lenartowicz, H. J. Yeh et al., "Functional connectivity of hippocampal networks in temporal lobe epilepsy," Epilepsia, vol. 55, no. 1, pp. 137-45, Jan, 2014.

[45] S. Dreifuss, F. J. Vingerhoets, F. Lazeyras et al., "Volumetric measurements of subcortical nuclei in patients with temporal lobe epilepsy," Neurology, vol. 57, no. 9, pp. 1636-41, Nov 13, 2001.

[46] M. M. Vandewouw, J. M. Young, M. M. Shroff et al., "Altered myelin maturation in four year old children born very preterm," Neuroimage Clin, vol. 21, pp. 101635, 2019. 